\title{
Somatotipo, Masa Grasa y Muscular del Escalador Deportivo Español de Elite
}

\author{
Somatotype, Fat and Muscle Mass of Elite Spanish Climbers \\ "José Ramón Alvero-Cruz; **Lluis Giner Arnabat; ${ }^{* * *}$ Fernando Alacid Cárceles; \\ ***** Miguel Ángel Rosety-Rodríguez \& ${ }^{* * * * *}$ Francisco Javier Ordóñez Muñoz
}

\begin{abstract}
ALVERO-CRUZ, J. R.; GINER, A. L.; ALACID C. F.; ROSETY-RODRÍGUEZ, M. A. \& ORDÓÑEZ, M. F. J. Somatotipo, masa grasa y muscular del escalador deportivo Español de elite. Int. J. Morphol., 29(4):1223-1230, 2011.

RESUMEN: Una adecuada morfología y composición corporal es una condición necesaria para obtener buenos resultados en el deporte de competición y una variable fundamental en el control del rendimiento deportivo. El objetivo del presente trabajo fue describir la masa grasa, la masa muscular y la morfología corporal de 11 escaladores deportivos de elite españoles, en base a métodos antropométricos para la composición corporal (ecuaciones de Faulkner, Carter y Durnin \& Womersley) y el somatotipo (Heath \& Carter) y de bioimp edancia eléctrica para la masa muscular (ecuaciones de Janssen \& Kyle). Un análisis de la varianza (ANOVA) fue aplicado para determinar las diferencias entre las diferentes fórmulas de cálculo del porcentaje de grasa. El porcentaje de grasa obtenido a partir de las ecuación de Durnin \& Womersley fue significativamente superior respecto al obtenido con la ecuación de Carter en hombres (7,51\% v/s 5,42\%) y al de Faulkner en mujeres $(14,40 \%$ v/s 12,58\%; p <0,05). La masa muscular esquelética fue superior en los hombres $(45,52 \%)$ que en las mujeres (34,28\%). Del somatotipo destacó el alto componente mesomórfico y la baja endomorfia en varones $(1,34$ - 5,22 - 3,05) y el morfotipo ectomesomorfo en mujeres (1,65 - 3,35 - 3,71). Los resultados del presente estudio pueden considerarse como valores de referencia en base al alto nivel deportivo de los sujetos estudiados.
\end{abstract}

PALABRAS CLAVE: Escalada deportiva; Somatotipo; Masa grasa; Masa muscular; Antropometría; Bioimpedancia eléctrica.

\section{INTRODUCCIÓN}

La escalada es una actividad deportiva que a principios de la década de los ochenta evolucionó hacia la competición en España. Al comienzo, las competiciones se realizaban en el medio natural, pero debido al impacto ambiental causado en la montaña (deportistas, público, transporte, materiales, etc.), hizo que la Federación Internacional replanteara ese modelo de competición para convertirlo en el actual, con eventos desarrollados sobre estructuras artificiales y a menudo en infraestructuras fijas ya existentes (Lloveras \& Alvesa, 2000). Actualmente, la escalada deportiva se desarrolla en 3 modalidades de competición, bien reglamentadas como son el Bloque, la Dificultad y la Velocidad.

Existen algunos trabajos que analizan la relación entre parámetros antropométricos en relación al rendimiento
(Mermier et al., 2000; Watts et al., 1993; Grant et al., 1996; Watts et al., 2003). Estos autores identifican a los escaladores deportivos como deportistas de baja estatura y porcentaje de grasa (Watts, 2004; España-Romero et al., 2009b), así como de una gran fuerza de prensión manual, índice de fuerza en relación al peso corporal (Watts), fuerza en el tronco y flexibilidad en la articulación de la cadera (Grant et al.). Sin embargo, Mermier et al. (2000) otorga a las variables antropométricas solamente un $0,3 \%$ de la varianza, que explicaría ese rendimiento, siendo el factor más importante, la propia actividad y el volumen de entrenamiento que explicaría un 58,9\% de la varianza. Otros aspectos destacados, enfatizan sobre la importancia de que el rendimiento en la escalada deportiva se basa en otros aspectos como la envergadura (Grant $e t$ al.) o la anchura y la masa libre de grasa

\footnotetext{
Departamento de Fisiología Humana y Educación Física y Deportiva. Escuela de Medicina de la Educación Física y el Deporte. Universidad de Málaga. España. Antropometrista Nivel 3 de ISAK.

** Director Técnico de FEDME (Federación Española Deportes de Montaña). España.

*** Departamento de Actividad Física y Deporte. Facultad de Ciencias del Deporte. Universidad de Murcia. España. Antropometrista Nivel 3 de ISAK.

***** Escuela de Medicina de la Educación Física y el Deporte. Universidad de Cádiz. España.

****** Dpto. de Anatomía y Embriología Humana. Universidad de Cádiz. España.
} 
del antebrazo (España-Romero et al., 2009c) y también en aspectos condicionales como la flexibilidad, la resistencia y la fuerza muscular tanto de tronco como de brazos y de manos (Grant et al.). La morfología y composición corporal son aspectos de fácil obtención e interpretación mediante técnicas como la antropometría y la bioimpedancia eléctrica. Por ello, el objetivo de este estudio fue describir los valores del somatotipo, grasa corporal y masa muscular esquelética, mediante ecuaciones antropométricas y de bioimpedancia eléctrica en escaladores deportivos españoles de élite.

\section{MATERIAL Y MÉTODO}

Once escaladores deportivos del equipo nacional español (8 varones y 3 mujeres) de edades comprendidas entre 20 y 30 años participaron en el estudio tras la firma del consentimiento informado y bajo los principios de la Declaración de Helsinki de 1961 así como de la Ley 14/2007, de 3 de julio, de Investigación biomédica (BOE, 159). Se obtuvieron las siguientes medidas antropométricas: peso; talla; pliegues cutáneos: tríceps, subescapular, bíceps, cresta iliaca, supraespinal, abdominal, anterior del muslo y medial de la pierna; perímetros corporales de brazo flexionado y contraído y pierna máxima; y diámetros biepicondíleo del húmero y bicondíleo del fémur. Todas las mediciones se realizaron siguiendo las normas estandarizadas de la Sociedad Internacional para el Avance de la Cineantropometría (ISAK) (Marfell-Jones et al., 2006). Bajo condiciones estandarizadas, se obtuvieron, valores de Impedancia (Z),
Resistencia (R), Reactancia (Xc) todos ellos en $\Omega$ y el ángulo de fase, con disposición de 4 electrodos (mano y pie derechos) (Lukaski et al., 1986, Alvero-Cruz et al., 2005).

Para la determinación del peso se utilizó una báscula SECA 803 (SECA, Alemania) de 100 g de precisión; para la estatura un tallímetro de pared SECA 206 (SECA, Alemania); para los pliegues cutáneos un plicómetro Holtain (Holtain Ltd, Crymych, Reino Unido) de 0,2 mm de precisión; para los perímetros una cinta métrica inextensible milimetrada Rosscraft (Rosscraft, Canadá); y para los diámetros, un paquímetro Holtain (Holtain Ltd, Crymych, Reino Unido), ambos instrumentos con una precisión de 0,1 cm. La bioimpedancia fue realizada con un Impedanciómetro Multifrecuencia Medisystem (Sanocare Human System SL, Madrid, Spain) sobre una camilla portátil plegable Ecopostural de superficie no metálica, utilizando electrodos desechables PKR415 (Sanocare Human System SL, Madrid, Spain). Entre las 8:00 y las 9:00 de la mañana, bajo condiciones de temperatura ambiente entre $22^{\circ}$ y $24^{\circ} \mathrm{C}$, en ayunas y tras orinar, se obtuvieron las medidas antropométricas y de impedancia eléctrica. Para esta última, los deportistas adoptaron la posición de decúbito supino durante 10 minutos, sobre una camilla de superficie no conductora, con ambas extremidades simétricas y a la misma altura, relajadas y en ligera abducción de $30^{\circ}$ a $45^{\circ}$. (Lukaski et al.). La bioimpedancia eléctrica efectuada fue, la convencional estándar, distal de cuerpo entero. Se procedió a la limpieza previa de la piel con alcohol y un total de cuatro electrodos fueron posicionados en los lugares estandarizados: dos electrodos en extremidad superior derecha (en cara dorsal y en posición de la articulación

Tabla I: Ecuaciones de estimación de la composición corporal por antropometría y bioimpedancia eléctrica.

\begin{tabular}{|c|c|c|c|}
\hline Técnica & Autor & Sexo & Ecuaciones de Estimación de la Composición Corporal \\
\hline \multirow[t]{6}{*}{ Antropometría } & Faulkner & $\mathrm{H}$ & $\%$ grasa: $(\Sigma 4 \mathrm{PLa} * 0,15)+5,783$ \\
\hline & Faulkner & M & $\%$ grasa: $(\Sigma 4 \mathrm{PLa} * 0,213)+7,9$ \\
\hline & Carter & $\mathrm{H}$ & $\%$ grasa: $2,585+\left(0,1051 \mathrm{x} \_6 \mathrm{PL}\right)$ \\
\hline & Carter & M & $\%$ grasa: $3,5803+(0,1548 \times$ x_6PL $)$ \\
\hline & Siri & $\mathrm{H}, \mathrm{M}$ & $\%$ grasa: $[(4,95 / \mathrm{Dc})-4,5] * 100$ \\
\hline & $\begin{array}{l}\text { Durnin \& } \\
\text { Womers ley }\end{array}$ & $\mathrm{H}, \mathrm{M}$ & $\mathrm{Dc}=1,1631-0,0632 * \log _{10}(-4 \mathrm{PLb})$ \\
\hline \multirow[t]{4}{*}{ BIA } & Kyle & $\mathrm{H}, \mathrm{M}$ & MLG $=-4,104+\left(0,518 * \mathrm{H}^{2} / \mathrm{R} 50\right)+(0,231 *$ Peso $)+(0,130 * \mathrm{Xc})+(4,229 *$ Sexo $)$ \\
\hline & Segal & $\mathrm{H}$ & MLG $=0,0006636 *\left(\mathrm{H}^{2}\right)-0,02117 *(\mathrm{R})+0,62854 *(\mathrm{P})-0,12380 *(\mathrm{E})+9,33285$ \\
\hline & Segal & M & MLG $=0,00064602 *\left(\mathrm{H}^{2}\right)-0,01397 *(\mathrm{R})+0,42087 *(\mathrm{P})+10,43485$ \\
\hline & Janssen & $\mathrm{H}, \mathrm{M}$ & Masa Muscular Esquelética $=\left[\left(\mathrm{Ht}_{2} / \mathrm{R} * 0,401\right)+\operatorname{Sexo} * 3,825+\right.$ Edad $\left.*-0,071\right]+5,102$ \\
\hline
\end{tabular}

H: Hombres, M: Mujeres; S4PLa : sumatorio de los pliegues : tríceps, subescapular, supraespinal y abdominal; S6PL: sumatorio de los pliegues : tríceps + subescapular + supraespinal + abdominal + muslo anterior + medial de la pierna; S4PLb: sumatorio de los pliegues tríceps, bíceps, subescapular y cresta iliaca; Dc: Densidad corporal; Ht2/R: Índice de impedancia (cm2/Ohm); R: Resistencia (Ohm); Xc: Reactancia (Ohm); MLG: Masa Libre de Grasa; Sexo: 1: Hombres, 0: Mujeres; H: Altura (m); P: Peso corporal (kg). 
carpo-radial y en el tercio distal del tercer hueso metacarpiano, también en su cara dorsal). Otros dos en extremidad inferior derecha (en la cara dorsal de la articulación tibio-tarsiana y en el tercer hueso metatarsiano). Previo a la realización del test, se procedió a la calibración mediante una resistencia externa conocida de $475 \Omega$, administrada por el fabricante, comprobando que el instrumento se encontraba adecuadamente calibrado (coeficiente de variación de $0,15 \%)$. Se indujo una corriente excitatoria de $800 \mathrm{~m} \AA$ a frecuencia conocidas de $1,10,25$, $50,75,100$ y $150 \mathrm{kHz}$, obteniéndose valores de Z, R, Xc y ángulo de fase, para cada una de las frecuencias.

La estimación de la composición corporal por ecuaciones derivadas a partir de pliegues cutáneos, han sido resumidas en la Tabla I. Se estimaron los porcentajes de grasa a partir de las ecuaciones de Faulkner (1968), Carter (1984) y Durnin \& Womersley (1974). El porcentaje de grasa a partir de valores de densidad corporal de la ecuación de Durnin \& Womersley, se estimó con la fórmula de Siri (1956).

La masa muscular esquelética se calculó a partir de la ecuación de Janssen et al. (2000) a partir de variables antropométricas y de bioimpedancia y la masa muscular apendicular por la ecuación de Kyle et al. (2003) que se refiere casi con exclusividad a la masa muscular existente en los miembros superiores e inferiores. Todos los cálculos en base a ecuaciones han sido realizados siguiendo las recomendaciones del Documento de Consenso de la Federación Española de Medicina del Deporte (Alvero-Cruz et al., 2010).

Se determinó el Índice de Masa Corporal (IMC) mediante la división de la masa corporal en kilogramos entre el cuadrado de la talla expresada en metros, así como los sumatorios de ocho y seis pliegues (eliminando del sumatorio de ocho pliegues los pliegues bíceps y cresta iliaca). Los componentes del somatotipo (endomorfia, mesomorfia y ectomorfia) y su análisis posterior fueron calculados mediante la metodología descrita por Carter \& Heath (1990).

Se realizó un estudio descriptivo de todas las variables (media y desviación típica), analizándose la normalidad de la muestra mediante el test de Kolmogorov-Smirnov.
El estudio de las diferencias entre las ecuaciones se realizó mediante el test de análisis de la varianza (ANOVA). Previo a este test se comprobó la igualdad de varianzas mediante el test de Levene y el posterior test no paramétrico de Student-Newman-Keuls. En todos los casos se consideraron valores significativos a un valor de $\mathrm{p}<0,05$, utilizando el paquete estadístico MedCalc 11.5.1.0 para Windows (Mariakerke, Belgium).

\section{RESULTADOS}

Las características de experiencia en la modalidad y entrenamiento de los escaladores se encuentran en la Tabla II. Los valores de peso, talla, IMC, así como en el sumatorio de 6 y 8 pliegues y valores de impedancia (Z), resistencia $(\mathrm{R})$, reactancia $(\mathrm{Zx})$ e índice de impedancia $(\mathrm{H} 2 /$ R) se encuentran en la Tabla III.

El somatotipo medio en los hombres fue de 1,345,22 - 3,05 y en mujeres de 1,65 - 3,35 - 3,71, para la endomorfia, mesomorfia y ectomorfia, respectivamente. Destacó la predominancia del componente mesomórfico en los hombres y el ectomórfico en las mujeres. Los hombres presentaron un índice de dispersión del somatotipo (SDI), entendido como la dispersión de los somatotipos individuales respecto al medio, de 2,58 reflejando una ligera heterogeneidad, mientras que en las mujeres el SDI fue de 1,67 reflejando una mayor homogeneidad en este grupo. En cuanto a los somatotipos medios se encontraron diferencias significativas entre las mujeres y los hombres presentando una distancia de dispersión del somatotipo de 4,75 y una distancia posicional somatotípica de 2,01 .

Los porcentajes de grasa corporal según cada una de las fórmulas estudiadas proporcionó valores significativamente diferentes en mujeres entre las ecuaciones de Faulkner y Durnin \& Womersley $(12,58 \%$ vs $14,40 \%$; p $<0,05)$. En los escaladores hombres se encuentran diferencias entre el porcentaje de grasa obtenido a partir de las ecuaciones de Carter y Durnin \& Womersley $(5,42 \%$ vs $7,51 \%$; p $<0,05)$ (Tabla IV).

Tabla II. Experiencia y características del entrenamiento de los escaladores.

\begin{tabular}{lcc}
\hline & Media & Des viación típica \\
\hline Entrenamiento (años) & 7,43 & 3,22 \\
Entrenamiento (días/semana) & 5,10 & 0,87 \\
Entrenamiento (horas/día) & 3,08 & 0,87 \\
Preparación física (horas/semana) (ciclismo, carrera, pesas, electroestimulación, etc.) & 2,82 & 3,45 \\
\hline
\end{tabular}


ALVERO-CRUZ, J. R.; GINER, A. L.; ALACID C. F.; ROSETY-RODRÍGUEZ, M. A. \& ORDÓÑEZ, M. F. J. Somatotipo, masa grasa y muscular del escalador deportivo Español de elite. Int. J. Morphol., 29(4):1223-1230, 2011.

Tabla III. Características antropométricas de la muestra

\begin{tabular}{llcccc}
\hline & & \multicolumn{2}{c}{ Hombres } & Mujeres \\
& & Media & Desviación típica & Media & Des viación típica \\
\hline Edad & $($ años $)$ & 27,90 & 2,00 & 27,60 & 0,57 \\
Peso & $(\mathrm{kg})$ & 59,50 & 4,83 & 46,76 & 6,70 \\
Talla & $(\mathrm{cm})$ & 168,50 & 5,26 & 158,90 & 7,00 \\
IMC & $\left(\mathrm{kg} \cdot \mathrm{m}^{-2}\right)$ & 20,92 & 1,48 & 18,52 & 1,17 \\
$\Sigma 6$ PL & $(\mathrm{mm})$ & 30,08 & 10,10 & 44,25 & 3,21 \\
$\Sigma$ 8 PL & $(\mathrm{mm})$ & 38,27 & 12,80 & 51,86 & 4,58 \\
$\mathrm{Z}$ & $(\Omega)$ & 498,33 & 47,02 & 628,16 & 52,50 \\
$\mathrm{R}$ & $(\Omega)$ & 481,08 & 46,07 & 617,07 & 53,48 \\
$\mathrm{Xc}$ & $(\Omega)$ & 127,70 & 17,81 & 109,03 & 7,21 \\
$\mathrm{H}^{2 /} \mathrm{R}$ & $(\Omega)$ & 59,05 & 5,50 & 40,91 & 7,08 \\
\hline
\end{tabular}

IMC: Índice de masa corporal; S6 PL: tríceps, subescapular, supraespinal, abdominal, anterior del muslo y medial de la pierna; S8 PL: tríceps, subescapular, bíceps, cresta iliaca, supraespinal, abdominal, anterior del muslo y medial de la pierna; Z: impedancia $(\Omega)$; R:Resistencia $(\Omega)$; Xc: Reactancia $(\Omega) ; \mathrm{Ht} 2 / \mathrm{R}$ : Índice de impedancia $(\Omega)$.

Tabla IV. Porcentajes de grasa y masa muscular por diversas ecuaciones antropométricas y de bioimpedancia eléctrica.

\begin{tabular}{|c|c|c|c|c|c|c|}
\hline \multirow[b]{2}{*}{ Compartimento } & & \multirow[b]{2}{*}{ Ecuación } & \multicolumn{2}{|r|}{ Hombres } & \multicolumn{2}{|r|}{ Mujeres } \\
\hline & & & Media & Desviación típica & Media & Desviación típica \\
\hline \multirow[t]{6}{*}{ Masa Grasa } & \multirow[t]{3}{*}{$\%$} & \multirow{3}{*}{$\begin{array}{l}\text { Faulkner } \\
\text { Carter } \\
\text { Dumin \& Womersley }\end{array}$} & $9,06^{*}$ & 0,96 & 12,58 & 0,73 \\
\hline & & & 5,42 & 0,88 & $9,96^{*}$ & 1,03 \\
\hline & & & 7,51 & 3,40 & 14,4 & 2,24 \\
\hline & \multirow[t]{3}{*}{$\mathrm{Kg}$} & Faulkner & 5,42 & 0,88 & 5,87 & 1,04 \\
\hline & & Carter & 3,68 & 0,91 & 4,62 & 0,89 \\
\hline & & Dumin \& Womersley & 4,54 & 2,21 & 6,85 & 1,88 \\
\hline \multirow[t]{5}{*}{ Masa Muscular } & \multirow[t]{2}{*}{$\%$} & Janssen & 45,52 & 1,86 & 34,28 & 1,1 \\
\hline & & Kyle & 17,76 & 2,10 & 13,66 & 0,42 \\
\hline & \multirow[t]{3}{*}{$\mathrm{Kg}$} & Janssen & 27,12 & 2,11 & 16,89 & 3,14 \\
\hline & & Kyle & 10,54 & 1,01 & 6,76 & 0,78 \\
\hline & & Ratio MM/ASMM & 2,60 & 0,33 & 2,49 & 0,18 \\
\hline
\end{tabular}

* Diferencias respecto al porcentaje de grasa según la ecuación de Durnin \& Womersley (p<0,05); Ratio MM/ ASMM: (Masa Muscular total/ Masa muscular apendicular).

La masa muscular esquelética en hombres obtuvo unos valores medios del $45,52 \%$ y para mujeres de un $34,28 \%$ y la relación de la masa muscular total y la masa muscular apendicular de las extremidades (ratio MM/ ASMM) entre hombres y mujeres presentó valores semejantes $(2,60$ vs $2,49 \mathrm{~kg})$.

\section{DISCUSIÓN}

Los deportistas integrantes de este estudio son considerados atletas de alto nivel dentro de la Federación Española de Deportes de Montaña y Escalada, de los cuales al- 
gunos de ellos han obtenido importantes éxitos deportivos en competiciones de máximo nivel internacional, por ello los valores antropométricos, podrían ser utilizados como referencia. Las variables más estudiadas por distintos autores, entre otras, han sido las antropométricas y su relación al rendimiento deportivo (Mermier et al., 2000; Watts et al., 1993; Grant et al.).

Es importante destacar la baja estatura y la pequeña masa corporal de los deportistas del presente estudio. Estos datos difieren de forma importante con los aportados por Mermier et al. (2000) que estudiaron a un grupo de 24 hombres escaladores con casi $10 \mathrm{~cm}$ más de talla media y superiores en $13 \mathrm{~kg}$ de peso. Sin embargo, en el estudio de Watts et al. (1993) el peso fue únicamente superior en $2 \mathrm{~kg}$, mientras que la talla fue de más de $10 \mathrm{~cm}$ por encima de la talla media de los escaladores del presente trabajo.

Entre los estudios realizados en escaladores hombres españoles encontramos cierta variabilidad en cuanto a la talla y el peso. Encontrando trabajos con valores similares en la estatura e inferiores en el peso $(171 \mathrm{~cm} \mathrm{y} 56 \mathrm{~kg}$ ) (Egocheaga et al., 1999), similar talla y mayor peso (172 cm y $66 \mathrm{~kg}$ ) (España-Romero et al., 2009c) y valores inferiores en ambas variables (163 cm y $56 \mathrm{~kg}$ ) (Muro et al., 1994). En las mujeres, la bibliografía aporta estudios en los que las escaladoras poseen un mayor talla y peso que las analizadas en este trabajo (162-163 cm y $51-53 \mathrm{~kg}$ ) (España-Romero et al., 2009c; Muro et al.). El peso del escalador es fundamental para conseguir subir de forma eficaz por vías verticales e incluso por zonas de verticalidad desplomada (Mermier et al., 2000; España-Romero et al., 2009b).

El IMC de nuestros escaladores es similar al reportado por Watts et al. (1993), encuadrándose los valores en percentiles considerados muy bajos (entre los percentiles 5 y 15) en las tablas de referencia consultadas (Must et al., 1991). La asociación del IMC con el rendimiento no suele ser habitual, sin embargo existe algún estudio realizado en escaladores de elite que aporta indicios de ello en mujeres, no hallándose esta asociación en la categoría masculina (España-Romero et al., 2009c).

La mayoría de estudios encontrados en la bibliografía arrojan datos de porcentaje de grasa corporal para hombres entre el 6,0 y el 9,8\%, estando en concordancia con los deportistas de este estudio (Mermier et al., 2000; Watts et al., 1993; Egocheaga et al.; España Romero et al., 2006, 2009a) (Tabla V). Valores más altos son los aportados por Grant et al. en escaladores con un de $14,0 \%$ de grasa y un gran peso corporal $(74 \mathrm{~kg})$ y en escaladores españoles con un $13,5 \%$, pero valorados mediante DEXA. (España Romero et al, 2009c). Esta diferencia existente entre los porcentajes estimados con ecuaciones de predicción de grasa corporal basadas en pliegues de grasa y el DEXA, normalmente con valores más altos en el DEXA ha sido descrita en adolescentes (Rodríguez et al., 2005). Los datos de nuestro estudio están en concordancia con los de datos obtenidos mediante la ecuación de Faulkner (Egocheaga et al.,) con valores en torno al 8,2\%. Otros datos similares a los aportados

Tabla V. Diferentes estudios de masa grasa en escaladores deportivos con diferentes métodos de estimación

\begin{tabular}{lccc}
\hline \multicolumn{1}{c}{ Referencia } & Ecuación y/o Método & Sexo & \% Masa Grasa * \\
\hline Grant et al. (1996) & Durnin \& Womersley & $\mathrm{H}$ & 14,0 \\
Mermier et al. (1997) & Durnin \& Womersley & $\mathrm{M}$ & 24,8 \\
& Pesaje Hidrostático & $\mathrm{H}$ & 6,8 \\
Egocheaga et al. (1999) & Pesaje Hidrostático & $\mathrm{M}$ & 14,6 \\
Mermier et al. (2000) & Faulkner & $\mathrm{H}$ & 8,2 \\
& Jackson \& Pollock & $\mathrm{H}$ & 9,8 \\
Watts (2004) & Jackson \& Pollock & $\mathrm{M}$ & 20,7 \\
& Jackson \& Pollock & $\mathrm{H}$ & 4,8 \\
Bertuzzi et al. (2007) & Jackson \& Pollock & $\mathrm{M}$ & 10,7 \\
España-Romero et al. (2006) & Jackson \& Pollock & $\mathrm{H}$ & 6,6 \\
& Jackson \& Pollock & $\mathrm{H}$ & 6,0 \\
España-Romero et al. (2009a) & Jackson \& Pollock & $\mathrm{M}$ & 12,0 \\
& Jackson \& Pollock & $\mathrm{H}$ & 6,2 \\
España-Romero et al. (2009c) & Jackson \& Pollock & $\mathrm{M}$ & 12,9 \\
& DEXA & $\mathrm{H}$ & 13,5 \\
\hline
\end{tabular}

* \% Masa Grasa: Se aportan valores medios de los estudios; H: hombres; M: mujeres. 
en nuestro estudio, fueron obtenidos mediante pesaje hidrostático (Mermier et al., 1997) y estimados con la ecuación de Jackson \& Pollock (De Moraes et al., 2007; España-Romero et al., 2006; 2009a).

En cuanto a las mujeres los porcentajes de grasa corporal oscilaron entre el 12,0 y 20,0\%, corroborándose valores más altos también por los aportados por Grant et al. $(24,8 \%)$ y por España Romero et al. (2009c) $(26,0 \%)$ mediante DEXA. Existe una tendencia, por parte de los escaladores de elite, de poseer un bajo porcentaje de grasa, ya que es considerado de gran importancia, puesto que un aumento de mismo supondría un esfuerzo muscular mayor durante los esfuerzos de progresión vertical (Watts).

La elección de las ecuaciones de masa grasa para este estudio se ven apoyadas por las recomendaciones del documento de Consenso del Grupo Español de Cineantropometría (GREC) (Alvero-Cruz et al., 2010) y en concreto las ecuación de Durnin \& Womersley, ya que fue descrita como la más precisa en este tipo de deportistas escaladores al ser comparada con un método de referencia en la estimación de la composición corporal como es el DEXA (España-Romero et al., 2009a), presentando solo un $-0,57 \%$ de diferencias intermétodos.

Pueden existir grandes variaciones en el cálculo de la masa muscular, bien si se hace por métodos antropomé-tricos, de impedancia eléctrica u otro tipo de procedimiento. La recogida de datos antropométricos no permitió estimar la masa muscular a partir de esas variables pero sí a partir de variables de impedancia eléctrica con la ecuación de Janssen et al. Estos valores del $45 \%$ en varones son concordantes con los aportados por Berral de la Rosa et al. (2010) con la estimación de las ecuaciones antropomé-tricas de Drinkwater (1984) o Kerr (1988), para jugadores de bádminton. Esto no significa que sea adecuado o no sea bien conocida la precisión real de esas ecuaciones. En mujeres, se ha estimado un valor medio del $34 \%$ del peso corporal, sin haber hallado en la bibliografía valores comparativos. Es destacable la igualdad entre varones y mujeres en el ratio MM/ASMM al relacionar la masa muscular total con la de miembros superiores e inferiores (Tabla IV).

El somatotipo de los hombres escaladores presentó un valor del componente mesomórfico medio-alto. En las mujeres existió un equilibrio entre los componentes mesomórfico y ectomórfico. Es de destacar no sólo las diferencias con el somatotipo medio femenino, sino con el estudio de Viviani \& Calderan (1991), en el que se estudió el somatotipo de 31 escaladores de nivel europeo obteniendo un somatotipo medio de 2,0-4,0-3,7. Estos valores presentan un componente endomórfico más alto y un componente mesomórfico más bajo que los aportados en el presente trabajo, pudiendo ser debido a que estos deportistas fueron estudiados en 1991 y probablemente la evolución de los métodos de entrenamiento y las tendencias actuales en el volumen de entrenamiento han podido producir aumentos importantes en el componente mesomórfico y cierta disminución del endomórfico.

En conclusión los escaladores deportivos se definen por poseer un somatotipo fundamentalmente mesomórfico en los varones y ectomesomórfico en las mujeres. Los porcentajes de grasa corporal se encuentran entre el 7,51\% y el $14,40 \%$ para hombres y mujeres, respectivamente. Mientras que la masa muscular esquelética en hombres fue del $45,52 \%$ en hombres y del $34,28 \%$ en mujeres de la masa corporal total.

\section{AGRADECIMIENTOS}

A la Federación Española de Montañismo y Escalada (FEDME) y a los integrantes del equipo español de Escalada Deportiva

ALVERO-CRUZ, J. R.; GINER, A. L.; ALACID C. F.; ROSETY-RODRÍGUEZ, M. A. \& ORDÓÑEZ, M. F. J. Somatotype, fat and muscle mass of elite Spanish climbers. Int. J. Morphol., 29(4):1223-1230, 2011.

SUMMARY: Somatotype and body composition is a necessary assessment in sports competition and training control. The purpose of this work was to determine the fat mass, the muscle mass and anthropometric somatotype of 11 sport climbers by means of anthropometric methods for body composition (Faulkner, Carter and Durnin \& Womersley algorithms), anthropometric somatotype (Heath-Carter), and bioelectrical impedance analysis for muscle mass (Janssen and Kyle formulae). Analysis of variance (ANOVA) was performed to determine differences among fat percentages. Fat percentage obtained via Durnin \& Womersley's equation was different versus Carter's equation in males $(7.51 \%$ v/s $5.42 \%)$ meanwhile in females this percentage was different respect to Faulkner's equation $(14.4 \%$ versus $12.58 \% ; \mathrm{p}<0.05)$. Muscle mass percentage in males $(45.52 \%)$ was higher than in females $(34.28 \%)$. In males the somatotype was endo-mesomorphic (1.34 - 5.22 - 3.05), whereas females were best described as mesoectomorphic (1.65 - 3.35 - 3.71$)$. These results can be considered as a reference values in body composition and somatotype in elite climbers.

KEY WORDS: Climbing, somatotype; Fat mass; Muscle mass; Anthropometry; Bioelectrical impedance. 


\section{REFERENCIAS BIBLIOGRÁFICAS}

Alvero-Cruz J. R.; Diego Acosta A. M.; Fernández Pastor V. J. \& García Romero J. Métodos de evaluación de la composición corporal: tendencias actuales (II). Archivos Medicina del Deporte, 12(105):43-9, 2005.

Alvero-Cruz, J. R.; Cabañas Armesilla, M. D.; Herrero de Lucas, A.; Martínez Riaza, L.; Moreno Pascual, C.; Porta, M. J.; Sillero, Q. M. \& Sirvent, B. J. E. Protocolo de valoración de la composición corporal para el reconocimiento médico-deportivo. Documento de consenso del Grupo español de cineantropometría (GREC) de la Federación Española de medicina del deporte (FEMEDE). Versión 2010. Archivos de Medicina del Deporte, 139:330-44, 2010.

Berral de la Rosa, F. J.; Rodríguez-Bies, E. C.; Berral de la Rosa, C. J.; Rojano Ortega, D. \& Lara Padilla, E. Comparación de ecuaciones antropométricas para evaluar la masa muscular en jugadores de badminton. Int. J. Morphol., 28(3):803-10, 2010.

De Moraes R. C.; Franchini B. E.; Kokubun E.; Augusta M. \& Dal Molin Kiss, P. Energy system contributions in indoor rock climbing. Eur. J. Appl. Physiol., 101:293-300, 2007.

Carter, J. E. L. Somatotype of Olympic Athletes from 1948 to 1976. En: Carter J.E.L. (ed.) Physical Structure of Olympic Athletes. Part II. Basel. Karger, 1984. pp, 80-109.

Carter, J. E. \& Heath, B. H. Somatotyping: Development and applications. $1^{\text {a }}$ ed. New York, Cambridge University Press, 1990.

Drinkwater, D. T. An anatomically derived method for the anthropometric estimation of human body composition. $\mathrm{Ph} \mathrm{D}$. Thesis, Simon Fraser University, Canada, 1984.

Durnin J. \& Womersley J. Body fat assessed from total body density and its estimation from skinfold thickness: measurements on 481 men and women aged from 16 to 72 years. Br. J. Nutr., 32:77-97, 1974

Egocheaga, J.; González, V.; Montoliu, M. A.; del Valle, M. \& Mendez, B. Condición física en escaladores deportivos de élite. Arch. Med. Deporte, 71:221-6, 1999.

España Romero, V.; Ortega Porcel, F.; García-Artero, E.; Ruiz, J. R. \& Gutiérrez Sainz, A. Performance, anthropometric and muscle strength characteristics in Spanish elite rock climbers. Selección. Revista Española e Iberoamericana de Medicina de la Educación Física y el Deporte, 15:176-83, 2006.

España-Romero, V.; Ruiz, J. R.; Ortega, F. B.; Artero, E. G.; Vicente, G.; Moreno, L. A.; Castillo, M. J. \& Gutierrez, A. Body fat measurement in elite sport climbers: Comparison of skinfold thickness equations with dual energy X-ray absorptiometry. $J$. Sports. Sci., 27(5):469-77, 2009a.
España Romero, V.; Artero, E. G.; Ortega, F. B.; Jiménez Pavón, D.; Gutiérrez, A.; Castillo, M. J. \& Ruiz, J. R. Aspectos fisiológicos de la escalada deportiva. Rev. Int. Med. Cienc. Act. Fis. Deporte, 35:264-98, 2009b.

España-Romero, V.; Ortega Porcel, F. B.; Artero, E. G.; JiménezPavón, D.; Gutiérrez Sainz, A.; Castillo Garzón, M. J. \& Ruiz, J. R. Climbing time to exhaustion is a determinant of climbing performance in high-level sport climbers. Eur. J. Appl. Physiol., $107: 517-25,2009 \mathrm{c}$

Faulkner J. A. Physiology of swimming and diving. En: Falls Exercise Physiology. Baltimore. Academic Press, 1968.

Grant, S.; Hynes, V.; Whittaker, A. \& Aitchison, T. Anthropometric strength endurance and flexibility characteristics of elite and recreational climbers. J. Sports. Sci., 14(4):301-9, 1996.

Janssen, I.; Heymsfield, S. B.; Baumgartner, R. N. \& Ross, R. Estimation of skeletal muscle mass by bioelectrical impedance analysis. J. Appl. Physiol., 89:465-71, 2000.

Kerr, D. A. An anthropometric method for fractionation of skin, adipose, bone, muscle and residual tissue masses in males and females age 6 to 77 year. Master Thesis, Simon Fraser University, Canada, 1988.

Kyle, U. G.; Genton, L.; Hans, D. \& Pichard, C. Validation of a bioelectrical impedance analysis equation to predict appendicular skeletal muscle mass (ASMM). Clin. Nutr., 22(6):537-43, 2003.

Lloveras, P. \& Alvesa, C. Bases para el entrenamiento en escalada. Madrid, Ed. Desnivel, 2000.

Lukaski, H. C.; Bolonchuck, W. W.; Hall, C. B. \& Siders, W. A. Validation of tetrapolar bioelectrical impedance method to assess human body composition. J. Appl. Physiol., 60:132732, 1986

Marfell-Jones M.; Olds, T.; Stewart, A. \& Carter, L. International standards for anthropometric assessment. Potchefstroom, South Africa, ISAK, 2006.

Mermier, C. M.; Robergs, R. A.; McMinn, S. M. \& Heyward, V.H. Energy expenditure and physiological responses during indoor rock climbing. Br. J. Sports. Med., 31:224-8, 1997.

Mermier, C. M.; Janot, J. M.; Parker, D. L. \& Swan, J. G. Physiological and anthropometric determinants of sport climbing performance. Br. J. Sports. Med., 34:359-65, 2000.

Muro, I.; Vila, R.; Vives, J. \& Gutiérrez, J. A. Estudio médicodeportivo de la escalada deportiva. Apunts. Medicina de l'Esport., 31:141-8, 1994. 
ALVERO-CRUZ, J. R.; GINER, A. L.; ALACID C. F.; ROSETY-RODRÍGUEZ, M. A. \& ORDÓÑEZ, M. F. J. Somatotipo, masa grasa y muscular del escalador deportivo Español de elite. Int. J. Morphol., 29(4):1223-1230, 2011.

Must A.; Dallal G.E. \& Dietz W.H. Reference data for obesity: 85th and 95th percentiles of body mass index (wt/ht2) and triceps skinfold thickness - a correction. Am. J. Clin. Nutr., 54:773, 1991.

Rodriguez, G.; Moreno, L. A.; Blay, M. G.; Blay, V. A.; Fleta, J.; Sarria, A.; Bueno, M. \& AVENA-Zaragoza Study Group.Body fat measurement in adolescents: comparison of skinfold thickness equations with dual-energy X-ray absorptiometry. Eur. J. Clin. Nutr., 59:1158-66, 2005.

Siri, W. E. Gross composition of the body. In: Advances in Biological and Medical Physics. Lawrence J. E. \& Tobias C.A.; New York, Academic Press, 1956. pp. 239-80.

Viviani F. \& Calderan M. The somatotype in a group of "top" free climbers. J. Sports. Med. Phys. Fitness, 31:581-6, 1991.

Watts, P. B; Martin, D. T. \& Durtschi, S. Anthropometric profiles of elite males and females competitive sport rock climbers. $J$. Sports. Sci., 11:113-7, 1993.

Watts, P. B.; Joubert, L. M.; Lish, A. K.; Mast, J. D. \& Wilkins, B. Anthropometry of young competitive sport rock climbers. $B r$. J. Sports. Med., 37:420-4, 2003.

Watts, P. B. Physiology of difficult rock climbing. Eur. J. Appl. Physiol., 91:361-72, 2004.

\author{
Dirección para correspondencia: \\ Dr. José Ramón Alvero Cruz \\ Escuela de Medicina de La Educación Física y el Deporte \\ Edifício López de Peñalver \\ Campus de Teatinos \\ Universidad de Málaga \\ 29071 Málaga, \\ ESPAÑA
}

E-mail: alvero@uma.es

Recibido : 26-04-2011

Aceptado: 28-07-2011 